\title{
Inverse limit spaces of post-critically finite tent maps
}

\author{
by
}

\author{
Henk B r u in (Pasadena, CA)
}

\begin{abstract}
Let $(I, T)$ be the inverse limit space of a post-critically finite tent map. Conditions are given under which these inverse limit spaces are pairwise nonhomeomorphic. This extends results of Barge \& Diamond [2].
\end{abstract}

1. Introduction. For a continuous self-map $f$ of a compact connected metric space $X$, one can build the inverse limit space as the space consisting of all inverse orbits:

$$
\left\{x=\left(\ldots, x_{-2}, x_{-1}, x_{0}\right): x_{i} \in X \text { and } x_{i}=f\left(x_{i-1}\right) \text { for all } i \leq 0\right\} \text {, }
$$

endowed with the metric $d(x, y)=\sum_{i<0} 2^{i}\left|x_{i}-y_{i}\right|$. An inverse limit space is a continuum, i.e., a compact connected metric space. There exists a natural homeomorphism $\widehat{f}$, namely $\widehat{f}\left(\left(\ldots, x_{-2}, x_{-1}, x_{0}\right)\right)=\left(\ldots, x_{-2}, x_{-1}, x_{0}, f\left(x_{0}\right)\right)$. This map is called the induced homeomorphism. The inverse of $\widehat{f}$ is the right shift. i.e.,

In this paper we will consider tent maps $T_{\alpha}:[0,1] \rightarrow[0,1]$ with slope $\alpha$,

$$
T_{\alpha}(x)= \begin{cases}\alpha x & \text { if } x \leq 1 / 2, \\ \alpha(1-x) & \text { if } x>1 / 2 .\end{cases}
$$

These maps have a unique turning or critical point $1 / 2$, henceforth denoted as $c$. Let $c_{i}=T^{i}(c)=T \circ \ldots \circ T(c)$ be the $i$ th iterate of the critical point. We assume that the critical orbit is a finite set, i.e., the critical point is periodic or strictly preperiodic. We will restrict $T$ to the core $I=\left[c_{2}, c_{1}\right]$.

2000 Mathematics Subject Classification: Primary 37B45; Secondary 54H20, 37C70, 37E05.

Key words and phrases: inverse limit space, interval map.

HB was supported by the Göran Gustafsson Foundation. This paper was written during the author's stay at Kungl. Tekniska Högskolan, Stockholm. The hospitality of the University of Rome II (Tor Vergata) where part of the research was done is also gratefully acknowledged. 
Inverse limit spaces often show up as attracting or repelling sets in dynamical systems. For example, the inverse limit space of the full tent map $T_{2}$ is homeomorphic to the closure of the unstable manifold of the fixed point of Smale's horseshoe. Barge \& Holte [3] showed that more general horseshoes, within the Hénon family, are homeomorphic to the inverse limit spaces we are discussing in this paper. More precisely, suppose $f_{a}(x)=1-a x^{2}$ is a quadratic map with a periodic turning point and $H_{a, b}(x, y)=\left(1-a x^{2}+y, b y\right)$ with the same value of $a$. Then for $b<0$ sufficiently close to 0 , there exists an open disk $V,[-1,1] \times\{0\} \subset V \subset \mathbb{R}^{2}$, such that $\operatorname{cl}_{a, b}^{n}(V) \subset V$ and $\bigcap_{n} H_{a, b}^{n}(V)$ is a space homeomorphic to one of the inverse limit spaces under consideration.

The purpose of this paper is to classify these inverse limit spaces as topological spaces. A classification of inverse limit spaces of so-called "hat-maps" (multiple one-dimensional horseshoes) was given in [16]. The inverse limit space of $T_{2}$ is the Knaster continuum. This space has been studied extensively. Bandt [1] showed that all composants are pairwise homeomorphic (except for the 0-composant). Another goal may be to classify the automorphisms (up to isotopy) of these inverse limit spaces. Fokkink [10, Chapter 2, Theorem 3.3] showed that the only automorphisms on the Knaster continuum are isotopic to iterates of the induced homeomorphism.

It is well known that the slope of $T_{\alpha}$ is determined by the dynamics of $T_{\alpha}$. To be precise, topological entropy is $h_{\text {top }}\left(T_{\alpha}\right)=\max \{\log \alpha, 0\}$. In this paper we extend a result of Barge and Diamond [2] which showed, among other things, that the inverse limit spaces of periodic tent maps $T_{\alpha}$ and $T_{\widetilde{\alpha}}$ are nonhomeomorphic whenever $\mathbb{Q}(\alpha)$ and $\mathbb{Q}(\widetilde{\alpha})$ are different algebraic extensions. We prove

TheOREM 1 (Main). Let $T_{\alpha}$ and $T_{\widetilde{\alpha}}$ be tent maps with finite critical orbits. If $\log \alpha$ and $\log \widetilde{\alpha}$ are rationally independent, then $\left(I, T_{\alpha}\right)$ and $\left(I, T_{\widetilde{\alpha}}\right)$ are not homeomorphic.

It was shown in [5] that if $T_{\alpha}$ has an $n$-periodic point, then $\left(I, T_{\alpha}\right)$ and $\left(I, T_{\widetilde{\alpha}}\right)$ can only be homeomorphic if the critical point of $T_{\widetilde{\alpha}}$ is also $n$-periodic. Indeed, the inverse limit spaces $\left(I, T_{\alpha}\right)$ and $\left(I, T_{\widetilde{\alpha}}\right)$ have the same number of endpoints only under this hypothesis.

The proof of the main theorem has some of the flavour of Watkins' proof [16]. A key ingredient in our proof are certain substitution systems, which describe the way how composants of $\left(I, T_{\alpha}\right)$ are folded. Substitutions are partially described by their associated matrices. Looking at these matrices only, and ignoring the additional structure of the substitutions, may lead to results similar to those of Barge and Diamond [2] (recently extended by Swanson and Volkmer [15]). In [11], Kailhofer presents a different approach 
to the folding patterns of the composants of $\left(I, T_{\alpha}\right)$ and obtains results of a more combinatorial flavour than Theorem $1\left({ }^{1}\right)$.

Acknowledgments. The author wants to thank the referee for the careful reading of the manuscript.

2. Chains and turnlinks. Throughout the paper $T$ is assumed to have a finite critical orbit. Therefore $\delta(T):=\min \left\{|x-y|: x \neq y \in \operatorname{orb}_{T}(c)\right\}>0$. It is well known that inverse limit spaces of tent maps are chainable (see e.g. [13]). A chain is a finite open cover $C=\left\{L_{i}\right\}_{i=1}^{N}$ of $(I, T)$ whose links $L_{i}$ and $L_{j}$ intersect if and only if $|i-j| \leq 1$. A space is said to be chainable if for every $\varepsilon>0$, there is a chain whose links have diameter less than $\varepsilon$.

Let us define a collection of natural chains. Let $Q_{t}=\left\{q_{t, 1}, q_{t, 2}, \ldots, q_{t, N(t)}\right\}$ $(t=0,1,2, \ldots)$ be points in the interval with the following properties:

- $c_{2}=q_{t, 1}<q_{t, 2}<\ldots<q_{t, N(t)}=c_{1}$.

- $\left\{c, c_{1}, c_{2}\right\} \subset Q_{t}$ for all $t$.

- $T^{-1}\left(Q_{t}\right) \subset Q_{t+1}$.

- For every $t \geq 0$ and $1 \leq i \leq N(t),\left|T^{t}\left(q_{t, i}\right)-T^{t}\left(q_{t, i+1}\right)\right| \leq 2^{-t} \eta$, where $0<\eta<\frac{1}{2} \delta(T)$.

Let $C_{t}$ be the chain whose links are given by

$$
L_{t, i}=\left\{x \in(I, T): x_{-t} \in\left(q_{t, i-1}, q_{t, i+1}\right)\right\}
$$

Here we adopt the convention that $L_{t, 1}=\left\{x \in(I, T): x_{-t} \in\left[c_{2}, q_{t, 2}\right)\right\}$ and $L_{t, N(t)}=\left\{x \in(I, T): x_{-t} \in\left(q_{t, N(t)-1}, c_{1}\right]\right\}$. Obviously, $C_{t}$ are chains and the diameters of the links tend to 0 as $t \rightarrow \infty$. By choosing $\eta$ small, the chains can be made as fine as required. Note that if we use these chains, chainability of $(I, T)$ is immediate.

If $C$ and $C^{\prime}$ are chains, $C$ is called finer than $C^{\prime}$ if for every link $L \in C$ there is a link $L^{\prime} \in C^{\prime}$ containing $L$. By construction, $C_{s}$ is finer than $C_{t}$ whenever $s \geq t$.

Definition 1. A link $L \in C$ is a turnlink if there exists an adjacent link $M \in C$, a chain $C^{\prime}=\left\{L_{j}^{\prime}\right\}_{j=1}^{N^{\prime}}$ and integers $a, b, 1 \leq a<b \leq N^{\prime}$, such that

- $L \cup M \supset \bigcup_{j=a}^{b} L_{j}^{\prime}$,

- $L \cap\left(\bigcup_{j=a}^{b} L_{j}^{\prime}\right) \neq \emptyset$,

- $L_{a}^{\prime}, L_{b}^{\prime} \subset M \backslash L$.

We say that $C^{\prime}$ turns in $L$ in this case. The link $L$ is an essential turnlink if every sufficiently fine chain $C^{\prime}$ has a turnlink in $L$.

$\left({ }^{1}\right)$ After this paper was submitted both L. Kailhofer and B. Raines have made claims that all nonconjugate tent maps with periodic critical points have nonhomeomorphic inverse limit spaces. 
Note that this definition involves arbitrary chains, not necessarily the natural chains introduced above. The first and last link of a chain is always a turnlink (e.g., the natural chain $C_{t+2}$ turns in $L_{t, 1}$ and $L_{t, N(t)} \in C_{t}$ ), but need not be an essential turnlink.

Write $\pi_{t}:(I, T) \rightarrow I$ for the projection to the $(-t)$ th component. The omega limit set of $x$ is the set of accumulation points of the orbit of $x$, i.e., $\omega(x):=\omega_{T}(x):=\bigcap_{i \geq 0} \overline{\bigcup_{j \geq i} T^{j}(x)}$.

Lemma 1. The link $L \in C_{t}$ is an essential turnlink if and only if $\omega(c) \cap$ $\pi_{t}(L) \neq \emptyset$.

Note that because orb $(c)$ is finite, $\omega(c)$ is nothing else than the periodic orbit that $c$ belongs to or is eventually mapped on.

Proof of Lemma 1. " $\Leftarrow$ " Suppose that $c_{n} \in \pi_{t}(L) \cap \omega(c)$. As $c_{n} \in \omega(c)$, there is a point $x=\left(\ldots, x_{-1}, x_{0}\right) \in L$ such that $x_{-t}=c_{n}$ and $x_{i} \in \omega(c)$ for all $i \leq 0$. Let $C^{\prime}$ be any chain refining $C_{t}$ and let $L^{\prime} \in C^{\prime}$ be such that $x \in L^{\prime} \subset L$. We will prove that $L^{\prime}$ is a turnlink.

Let $\varepsilon=\sup \left\{d(y, z): y, z \in L^{\prime}\right\}$. Assuming that $C^{\prime}$ is sufficiently fine, $\varepsilon<\eta<\frac{1}{2} \delta(T)$. Take $s$ so large that $C^{\prime \prime}:=C_{s}$ refines $C^{\prime}$ and $2^{-s}<\varepsilon$. Moreover, choose $s$ such that $c_{s}=c_{n}$. Then there exist $a<b$ such that $J:=\pi_{s}\left(\bigcup_{j=a}^{b} L_{j}^{\prime \prime}\right)$ contains $c$ and is a maximal interval on which $T^{s} \mid J$ has a single turning point (namely $c$ ). Then $\pi_{0}\left(\bigcup_{j=a}^{b} L_{j}^{\prime \prime}\right)$ is an interval stretching from $c_{n}$ to another point $u \in \operatorname{orb}(c)$ satisfying $u \in \operatorname{cl} \pi_{0}\left(L_{a}^{\prime \prime}\right) \cap \operatorname{cl} \pi_{0}\left(L_{b}^{\prime \prime}\right)$. Moreover, for any two points $y, z \in \bigcup_{j=a}^{b} L_{j}^{\prime \prime}$ such that $\pi_{0}(y)=\pi_{0}(z)$ we have $d(y, z)<\varepsilon$. It follows that $C_{s}$ turns in $L^{\prime}$.

" $\Rightarrow$ " Let $p>c$ be the orientation reversing fixed point of $T$. Let us start by presenting the arc component $P$ through $\bar{p}=(\ldots, p, p, p)$. This is the largest arc connected set containing $\bar{p}$. It can be written as $\bigcup_{n} P_{n}$ where $P_{n}=\left\{x: x_{i} \geq c\right.$ for all $\left.i<-n\right\}$. Because $P_{n}$ can be parametrized by its $(-n)$ th component, it is an arc. The union $P$ is clearly dense in $(I, T)$.

Assume now that $L^{\prime} \in C^{\prime}, L^{\prime} \subset L$, is a turnlink, and assume that $\left\{L_{j}^{\prime \prime}\right\}_{j=a}^{b}$ turns in $L^{\prime} \cup M^{\prime}$. Here $M^{\prime}$ is the appropriate link adjacent to $L^{\prime}$. The arc component $P$ lies dense in $(I, T)$. In particular, there is an arc $A \subset P$ such that $A \subset \bigcup_{j=a}^{b} L_{j}^{\prime \prime}$ and $A \cap L_{j}^{\prime \prime} \neq \emptyset$ for all $a \leq j \leq b$. This implies that also $\pi_{t}(A)$ must turn in $\pi_{t}\left(L^{\prime} \cup M^{\prime}\right)$. But since $A \subset P_{n}$ for some $n \geq t, \pi_{t}(A)$ can only turn if $\pi_{t}\left(P_{n}\right)$ turns, i.e., if it contains the image of a turning point of $T^{n-t}$. This is a point $y=T^{i}(c)$ for some $1 \leq i \leq n-t$, and there are points $x_{1}, x_{2}, x_{3} \in P_{n}$ such that

- $\pi_{t}\left(x_{2}\right)=y$.

- $\pi_{t}\left(x_{1}\right)=\pi_{t}\left(x_{3}\right)=y \pm \eta$, where as before $0<\eta<\frac{1}{2} \delta(T)$.

- $\pi_{t}(z) \neq y \pm \eta$ for any $z \in P_{n}$ strictly between $x_{1}$ and $x_{3}$. 
- $x_{1}, x_{3} \in N^{\prime} \in C^{\prime}$. Here we assume for simplicity that $C^{\prime}=C_{s}$ for some large $s$.

It follows that $\left(\pi_{t+i}\left(x_{1}\right), \pi_{t+i}\left(x_{3}\right)\right)$ is an interval containing the critical point, and $\left|\pi_{t+i}\left(x_{1}\right)-\pi_{t_{i}}\left(x_{3}\right)\right| \geq \eta / \alpha^{i}$, where $\alpha$, the slope of $T$, serves as Lipschitz constant. Therefore $d\left(x_{1}, x_{3}\right) \geq 2^{-i} \eta / \alpha^{i}$. On the other hand, the chain $C^{\prime}$ can be taken arbitrarily fine and $N^{\prime}$ arbitrarily small. As $x_{1}, x_{3} \in N^{\prime}$, $i$ becomes arbitrarily large and consequently $y \in \omega(c)$.

This lemma seems to indicate that chains have precisely \# $\#(c)$ turnlinks. This is not completely true, because two adjacent links may both be turnlinks with respect to the same $y \in \omega(c)$. Hence turnlinks may come in pairs, but there are precisely $\# \omega(c)$ "clusters" of essential turnlinks.

LEMmA 2. If $L \in C_{t}$ is a turnlink, then $\pi_{t}(L)$ contains a point in $\bigcup_{i \geq 1} T^{i}(c)$.

Proof of Lemma 2. This is just the second part of Lemma 1, except that, because the turnlink $L$ need not be essential, we cannot take the chain $C^{\prime}$ arbitrarily small. Therefore we can only conclude that $\pi_{t}(L) \cap \bigcup_{i \geq 1} T^{i}(c)$ $\neq \emptyset$.

Hence, if $c$ is periodic, then the turnlinks of $C_{t}$ coincide with the essential turnlinks of $C_{t}$. If $c$ is strictly preperiodic, this need not be true.

COROLlary 1. If $T$ and $\widetilde{T}$ are tent maps such that $\omega_{T}(c)$ and $\omega_{\widetilde{T}}(c)$ have different cardinality, then the inverse limit spaces $(I, T)$ and $(I, \widetilde{T})$ are not homeomorphic.

This extends a result of Barge and Martin [5], showing the same thing for maps with a periodic critical point.

Proof of Corollary 1. Suppose that $\# \omega_{T}(c)<\# \omega_{\widetilde{T}}(c)$. Let $h:(I, T) \rightarrow$ $(I, \widetilde{T})$ be a homeomorphism. Take $t$ so large that $h\left(C_{t}\right)$ refines $\widetilde{C}_{0}$. Clearly, $h(L)$ is contained in an essential turnlink of $\widetilde{C}_{0}$ whenever $L \in C_{t}$ is an essential turnlink. By the pigeonhole principle (and the choice of $\eta$ ) there is at least one essential turnlink $\widetilde{L} \in \widetilde{C}_{0}$ left out. Take some chain $\widetilde{D}$ refining $h\left(C_{t}\right)$ which turns in $\widetilde{L}$. Because the turnlink $\widetilde{L}$ is essential, such a chain $\widetilde{D}$ can be found. Then $h^{-1}(\widetilde{D})$ is a chain turning in some link in $h^{-1}(\widetilde{L})$. But $h^{-1}(\widetilde{L})$ is disjoint from the essential turnlinks of $C_{t}$. This contradicts Lemma 1.

Using limits of turnlinks one can show that inverse limit spaces of tent maps are not homogeneous. Indeed, let us make the following definition:

Definition 2. A point $q \in(I, T)$ is a turnlink point if for every neighbourhood $U \ni q$, every sufficiently fine chain has a turnlink (and therefore essential turnlink) in $U$. 
If $\omega(c)$ is a periodic orbit $\operatorname{orb}(p)=\left\{p_{1}, p_{2}, \ldots, p_{n}\right\}$, then the techniques from Lemma 1 can be used to show that there are only $n$ turnlink points: $\left(\ldots, p_{n}, p_{1}, p_{2}, \ldots, p_{n}\right)$ and its shifts. If $c$ is $n$-periodic, these points are actually the $n$ endpoints of $(I, T)$; see [5].

A turnlink point $q$ can be either one-sided or two-sided. Assume that $C$ is a chain and the link $L$ containing $q$ is not the first or the last link. Then $q$ is one-sided if there is a single link $M$ adjacent to $L$ such that every sufficiently fine chain turns in $L \cup M$. If $M^{\prime}$ is the other adjacent link and sufficiently fine chains turn both in $L \cup M$ and in $L \cup M^{\prime}$, then $q$ is a two-sided turnlink point. If $c$ is periodic, then every endpoint as turnlink point is one-sided. If $c$ is strictly preperiodic, say $T^{t}(c)=T^{t+n}(c)=p$, then the turnlink points are one-sided if $p$ is orientation preserving and two-sided if $p$ is orientation reversing. If $c$ is strictly preperiodic, then (with the exception of the full tent map, whose inverse limit space is the standard Knaster continuum = bucket handle) the turnlink points are not endpoints.

A nice illustration of a two-sided turnlink point is the inverse limit space of the tent map with slope $\sqrt{2}$. In this case $c_{3}=p>c$ is the fixed point. The inverse limit space consists of two bucket handles glued together at their end-points (see [4]). The glue point becomes the (unique) turnlink point.

With the exception of the turnlink points, the inverse limit space of a post-critically finite tent map is locally homeomorphic to a Cantor set of arcs.

3. Maps with periodic turning points. Our next aim is to describe how $C_{t}$ coils through $C_{s}$ for $t \geq s$. We do this for the case when the critical point is periodic. At this point we will also assume that $\sqrt{2}<\alpha \leq 2$. It is well known that $T_{\alpha}$ is locally eventually onto in this case, i.e., for any nondegenerate interval $J \subset[0,1]$, we have $f^{i}(J) \supset\left[T_{\alpha}^{2}(c), T_{\alpha}(c)\right]$ for $i$ sufficiently large. The case $\alpha \leq \sqrt{2}$ will be discussed in Corollary 2 .

Let $c_{2}=y_{1}<\ldots<y_{n}=c_{1}$ be the critical orbit spatially ordered. Let $a_{i}, 1 \leq i \leq n-1$, denote the intervals $\left[y_{i}, y_{i+1}\right]$. Write

$$
\chi\left(a_{i}\right)= \begin{cases}a_{j} a_{j+1} \ldots a_{j+k} & \text { if } f:\left[y_{i}, y_{i+1}\right] \rightarrow\left[y_{j}, y_{j+k+1}\right] \text { o.p. } \\ a_{-j-k} \ldots a_{-j-1} a_{-j} & \text { if } f:\left[y_{i}, y_{i+1}\right] \rightarrow\left[y_{j}, y_{j+k+1}\right] \text { o.r. }\end{cases}
$$

Here o.p. and o.r. stand for orientation preserving and orientation reversing. In other words, the symbol $a_{-j}$ indicates $\left[y_{j}, y_{j+1}\right]$ traversed in the orientation reversing way. Consequently,

$$
\chi\left(a_{-i}\right)= \begin{cases}a_{j} a_{j+1} \ldots a_{j+k} & \text { if } f:\left[y_{i}, y_{i+1}\right] \rightarrow\left[y_{j}, y_{j+k+1}\right] \text { o.r. }, \\ a_{-j-k} \ldots a_{-j-1} a_{-j} & \text { if } f:\left[y_{i}, y_{i+1}\right] \rightarrow\left[y_{j}, y_{j+k+1}\right] \text { o.p. }\end{cases}
$$

The definition of $\chi$ extends to finite and infinite words by concatenation: if $s_{i}$ are symbols, then $\chi\left(s_{1} \ldots s_{n}\right)=\chi\left(s_{1}\right) \ldots \chi\left(s_{n}\right)$ and $\chi\left(s_{1} s_{2} \ldots\right)=$ $\chi\left(s_{1}\right) \chi\left(s_{2}\right) \ldots$ 
The word $a_{1} a_{2} \ldots a_{n-1}$ indicates the whole interval, as well as how $C_{t}$ coils through itself. The word $\chi\left(a_{1} a_{2} \ldots a_{n-1}\right)$ indicates how $T$ maps the interval onto itself, as well as how $C_{t}$ coils through $C_{t-1}$. In general, the word $\chi^{t-s}\left(a_{1} a_{2} \ldots a_{n-1}\right)$ indicates how $C_{t}$ coils through $C_{s}$.

As we shall see in the proof of Theorem 2, there is a symbol $\varrho_{1}$ such that $\chi^{n}\left(\varrho_{1}\right)$ starts with (and is longer than) $\varrho_{1}$. It follows that $\chi^{i}\left(\varrho_{1}\right)$ converges to an infinite $n$-periodic sequence $\varrho=\varrho_{1} \varrho_{2} \ldots$

Obviously, $\operatorname{orb}_{T}(c)$ defines a Markov partition for $T$, having an $(n-1)$ $\times(n-1)$ transition matrix $B$. The Perron-Frobenius Theorem applies to $B$; hence $B$ has a positive leading eigenvalue which has a larger absolute value than all other eigenvalues. It is well known that this leading eigenvalue equals $\exp \left(h_{\text {top }}\left(T_{\alpha}\right)\right)=\alpha$. Some of the properties of $\chi$ can be derived from the associated matrix $B^{\prime}=\left(b_{i, j}^{\prime}\right)$, where

$$
b_{i, j}^{\prime} \text { is the number of } \begin{cases}a_{j} \text { in } \chi\left(a_{i}\right) & \text { if } i, j \leq n-1, \\ a_{j} \text { in } \chi\left(a_{n-1-i}\right) & \text { if } j \leq n-1<i, \\ a_{n-1-j \text { in } \chi\left(a_{i}\right)} & \text { if } i \leq n-1<j, \\ a_{n-1-j} \text { in } \chi\left(a_{n-1-i}\right) & \text { if } n-1<i, j .\end{cases}
$$

This corresponds to arranging the symbols as $a_{1} a_{2} \ldots a_{n-1} a_{-1} a_{-2} \ldots$ $a_{-(n-1)}$. For more information on substitutions we refer to the monograph of Queffélec [14]. We will only need the following statements which follow easily from the Perron-Frobenius Theorem and elementary linear algebra. Write $\varrho=\varrho_{1} \varrho_{2} \ldots$ as above and let || denote the length of a string. Then

$$
\lim _{m \rightarrow \infty} \frac{\left|\chi^{n}\left(\varrho_{i+1} \ldots \varrho_{i+m}\right)\right|}{m} \rightarrow \text { leading eigenvalue of } B^{\prime n}
$$

and this convergence is uniform in $i$.

Lemma 3. Assume that \# $\operatorname{orb}_{T}(c)=n$ and let $B$ and $B^{\prime}$ be as above. Then $B^{\prime}$ has the eigenvalues of $B$ as well as $n-1$ eigenvalues (counted with multiplicity) on the unit circle. In particular, $B$ and $B^{\prime}$ have the same leading eigenvalue.

Proof. Assume that $c=y_{k+1}$. The matrix $B$ has a "unimodal" shape in the sense that $B=\left(\begin{array}{l}P \\ Q\end{array}\right)$ where $P$ is a $k \times(n-1)$ matrix whose ones run in a southeast direction, while $Q$ is a $(n-1-k) \times(n-1)$ matrix whose ones run from northeast to southwest. By (1), the matrix $B^{\prime}$ has the shape

$$
B^{\prime}=\left(\begin{array}{cc}
P & 0 \\
0 & Q \\
0 & P \\
Q & 0
\end{array}\right)
$$

To calculate $\operatorname{det}\left(B^{\prime}-\lambda I\right)$, first add column $n-1+i$ to column $i$ for $1 \leq i<n$, 
then subtract row $i$ from row $n-1+i$ for $1 \leq i<n$ to obtain

$\operatorname{det}\left(B^{\prime}-\lambda I\right)=\operatorname{det}\left(\begin{array}{cc}B-\lambda I & 0 \\ & P^{Q} \\ 0 & -Q^{-\lambda I}\end{array}\right)=\operatorname{det}(B-\lambda I) \operatorname{det}\left(\begin{array}{c}P \\ -Q\end{array}-\lambda I\right)$.

It remains to show that $\left(\begin{array}{c}P \\ -Q\end{array}\right)$ has only eigenvalues on the unit circle. Note that $\left(\begin{array}{c}P \\ -Q\end{array}\right)$ is the "signed" transition matrix of $T$ : a factor -1 is added whenever the transition from one state to another reverses orientation. Therefore the entries of $\left(\begin{array}{c}P \\ -Q\end{array}\right)^{m}$ represent the total number of transitions by $T^{m}$ from one state to another, orientation reversing transitions counted negative. Since $T$ is continuous, these entries are in $\{-1,0,1\}$ for all $m \geq 0$.

If $v$ is an eigenvector of $\left(\begin{array}{c}P \\ -Q\end{array}\right)$, and $\lambda,|\lambda|>1$, its eigenvalue, then $\left\|\left(\begin{array}{c}P \\ -Q\end{array}\right)^{m} v\right\|=|\lambda|^{m}\|v\| \rightarrow \infty$. However, the previous argument shows that $\left\|\left(\begin{array}{c}P \\ -Q\end{array}\right)^{m} v\right\|$ is bounded. A similar argument works for $0<|\lambda|<1$. It therefore remains to show that 0 is not an eigenvalue of $\left(\begin{array}{c}P \\ -Q\end{array}\right)$. Let us calculate $\operatorname{det}\left(\begin{array}{c}P \\ -Q\end{array}\right)$. Since $c_{2}$ is the global minimum and $T\left(c_{2}\right)>c_{2}$, the first column of $\left(\begin{array}{c}P \\ -Q\end{array}\right)$ consists of zeros and -1 at the bottom. Developing $\operatorname{det}\left(\begin{array}{c}P \\ -Q\end{array}\right)$ along this column gives

$$
\operatorname{det}\left(\begin{array}{c}
P \\
-Q
\end{array}\right)= \pm \operatorname{det}\left(\begin{array}{c}
P^{\prime} \\
-Q^{\prime}
\end{array}\right),
$$

where $\left(\begin{array}{c}P^{\prime} \\ -Q^{\prime}\end{array}\right)$ is a signed unimodal $(n-2) \times(n-2)$ transition matrix. Because $T$ is unimodal, either $T\left(y_{1}\right)=y_{2}\left(\right.$ and $\left.P_{1,2}^{\prime}=1\right)$ or $T\left(y_{n}\right)=y_{2}\left(\right.$ and $Q_{n-2-k, 2}=$ 1). Hence the first column of $\left(\begin{array}{c}P^{\prime} \\ -Q^{\prime}\end{array}\right)$ has precisely one nonzero entry. Repeating the argument gives $\operatorname{det}\left(\begin{array}{c}P \\ -Q\end{array}\right)= \pm 1 \neq 0$.

THEOREM 2. Let $T=T_{\alpha}$ and $\widetilde{T}=T_{\widetilde{\alpha}}$ be tent maps both having an $n$-periodic critical orbit. If $(\log \alpha) / \log \widetilde{\alpha} \notin \mathbb{Q}$, then the inverse limit spaces $(I, T)$ and $(I, \widetilde{T})$ are not homeomorphic.

It should be borne in mind that if $T^{k}$ and $\widetilde{T}^{l}$ (with $\alpha \neq \widetilde{\alpha}$ ) are topologically conjugate for some $k, l \geq 1$, then $(I, T)$ and $(I, \widetilde{T})$ are homeomorphic. Although the relation $T^{k}=\widetilde{T}^{l}$ can be shown to be false (as it would imply that $T$ and $\widetilde{T}$ have the same maximum), it should be no surprise that a condition like $\alpha^{k} \neq \widetilde{\alpha}^{l}$ turns up in this theorem.

Proof of Theorem 2. Suppose by contradiction that $h:(I, T) \rightarrow(I, \widetilde{T})$ is a homeomorphism. The idea is to compute, in two different ways, how $C_{s}$ coils through $C_{0}$ for $s$ large, and show that they cannot match. The direct way is given by applying $\chi^{s}$ to $a_{1} \ldots a_{n-1}$. The second way is by means of $h$. Take $t_{0}$ so large that $h^{-1}\left(\widetilde{C}_{t_{0}}\right)$ refines $C_{0}$. Since the $n$ essential turnlinks of 
$\widetilde{C}_{t_{0}}$ must be mapped into the $n$ essential turnlinks of $C_{0}, h^{-1}$ must map the partial chains $\left\{\widetilde{L}_{t_{0}, i}\right\}_{i=a}^{b}$ of $\widetilde{C}_{t_{0}}$ covering $\widetilde{a}_{j}$ (i.e., $\left[\widetilde{y}_{j}, \widetilde{y}_{j+1}\right] \subset \widetilde{\pi}_{t_{0}}\left(\bigcup_{i=a}^{b} \widetilde{L}_{t_{0}, i}\right)$ and $\widetilde{\pi}_{t_{0}}\left(\widetilde{L}_{t_{0}, i}\right) \cap\left[\widetilde{y}_{j}, \widetilde{y}_{j+1}\right] \neq \emptyset$ for $\left.a \leq i \leq b\right)$ to a union of similar partial chains of $C_{0}$. Therefore $h^{-1}$ can be represented by a substitution $\psi$, where

$$
\psi\left(\widetilde{a}_{j}\right)=a_{i_{1}} \ldots a_{i_{k}}
$$

when $\pi_{0} \circ h^{-1}\left(\left\{\widetilde{x} \in(I, \widetilde{T}): \widetilde{\pi}_{t_{0}}(\widetilde{x}) \in\left(\widetilde{y}_{j}, \widetilde{y}_{j+1}\right)\right\}\right)$ respectively covers $a_{i_{1}}, \ldots$ $\ldots, a_{i_{k}}$. Let now $t \gg t_{0}$ be arbitrary and $s$ so large that $h\left(C_{s}\right)$ refines $\widetilde{C}_{t}$. The chain $h\left(C_{s}\right)$ can only turn in turnlinks $L$ of $\widetilde{C}_{t}$ and crosses the other links transversally. Due to Lemma $2, \pi_{t}(L) \cap \bigcup_{i \geq 1} \widetilde{T}_{i}(c)=\pi_{t}(L) \cap \omega_{\widetilde{T}}(c) \neq \emptyset$, and because the turnlinks of $\widetilde{C}_{t}$ are the same as the essential turnlinks, we can represent the action of $h$ on $C_{s}$ by a substitution $\psi_{s, t}$. By construction, each link $\widetilde{T}^{t-t_{0}}(L), L \in \widetilde{C}_{t}$, is contained in a link of $\widetilde{C}_{t_{0}}$. In particular, $\widetilde{T}^{t-t_{0}}$ maps the turnlinks of $\widetilde{C}_{t}$ into turnlinks of $\widetilde{C}_{t_{0}}$. The coiling of $h\left(C_{s}\right)$ through $\widetilde{C}_{t_{0}}$ can therefore be written as $\widetilde{\chi}^{t-t_{0}} \circ \psi_{s, t}$, and the coiling of $C_{s}=h^{-1} \circ h\left(C_{s}\right)$ through $C_{0}$ as $\psi \circ \widetilde{\chi}^{t-t_{0}} \circ \psi_{s, t}$. Hence

$$
\chi^{s}=\psi \circ \widetilde{\chi}^{t-t_{0}} \circ \psi_{s, t} .
$$

Recall that $y_{1}$ is $n$-periodic and the global minimum of $T$. This shows that for some $y \in\left(y_{1}, y_{2}\right], T^{n}\left(\left[y_{1}, y\right]\right)$ covers $\left[y_{1}, y_{2}\right]$ in an orientation preserving way. From these two observations it follows that

$$
n=\min \left\{m: \chi^{m}\left(a_{1}\right) \text { starts with } a_{1}\right\} .
$$

This implies that $\chi^{s n}\left(a_{1}\right)$ converges to a fixed point $\varrho=\varrho_{1} \varrho_{2} \ldots=a_{1} \ldots$ of $\chi^{n}$. The same statement is true for $\widetilde{\chi}$.

Let $b_{s, t}$ be the first symbol of $\psi_{s, t}\left(a_{1} \ldots a_{n-1}\right)$. By adjusting $t_{0}$ if necessary and taking $t-t_{0}$ a multiple of $n$, we can assume that $b_{s, t} \equiv \widetilde{a}_{1}$. As $t$ can be taken arbitrarily large, it follows that

$$
\varrho=\psi(\widetilde{\varrho}) \text {. }
$$

Next we have to rule out that $\varrho$ is periodic under the shift $\sigma$. Since $T_{\alpha}$ is locally eventually onto, there exists $k$ such that $\chi^{k}\left(\varrho_{1}\right)$ contains all symbols. In other words, $\chi$ and its associated matrix $B^{\prime}$ are primitive. By the PerronFrobenius Theorem, $B^{\prime}$ has a positive left eigenvector $w$ associated with its leading eigenvalue $\alpha$; its components indicate the relative frequencies of the symbols $a_{j}$ in $\varrho$. If $\varrho$ is periodic under the shift, all of these frequencies are rational, and therefore $\alpha$ is an integer. Hence $\alpha=2$. The map $T_{2}$ indeed generates a periodic sequence $\varrho$, but the inverse limit space $\left(I, T_{2}\right)$ is clearly distinct from all other inverse limit spaces. So from now on assume that $\varrho$ and $\widetilde{\varrho}$ are not periodic under $\sigma$.

The fact that $\log \alpha$ and $\log \widetilde{\alpha}$ are rationally dependent follows immediately from a result of Durand (a special case of [9, Theorem 14]). In our 
setting we can also use the following argument. Given $m \geq 1$, let

$$
\kappa(m)=\min \left\{i \geq 1: \varrho_{i} \neq \varrho_{i+m}\right\},
$$

that is, $\kappa(m)$ indicates for how long $\sigma^{m}(\varrho)$ coincides with $\varrho$. As $\varrho$ is not periodic under $\sigma, \kappa(m)<\infty$ for all $m \geq 1$.

We say that $\varrho$ is asymptotically translation invariant over $v$ if there exists a sequence $\left\{m_{i}\right\}$ such that

$$
\left\{\begin{array}{l}
\log m_{i}-\log m_{i-1} \rightarrow v \text { exponentially fast, and } \\
\inf _{i} \kappa\left(m_{i}\right) / m_{i}>0 .
\end{array}\right.
$$

By the spectral properties of the matrix $B^{\prime}$ (see (2) and Lemma 3), $\varrho$ is asymptotically translation invariant over $n \log \alpha$. Indeed, the sequence $\left\{m_{i}\right\}$ with $m_{1}=\min \left\{i>1: \varrho_{i}=a_{1}\right\}-1$ and

$$
m_{i}=\left|\chi^{n}\left(\varrho_{1} \ldots \varrho_{m_{i-1}}\right)\right| \approx C_{0} \alpha^{i n}+\mathcal{O}\left(\alpha_{0}^{i n}\right)
$$

for some $C_{0}>0$ and $\alpha_{0}<\alpha$ satisfies (3). For $\alpha_{0}$ we can take the second largest eigenvalue of $B^{\prime n}$, which by the Perron-Frobenius Theorem is indeed strictly less than $\alpha$.

We claim that if $\varrho$ is asymptotically translation invariant over $v$ and $v^{\prime}$, then $v / v^{\prime}$ is rational. Indeed, let $\left\{m_{i}\right\}$ and $\left\{m_{j}^{\prime}\right\}$ satisfy (3) for $v$ and $v^{\prime}$ respectively. Let $\delta=\min \left\{\inf _{i} \kappa\left(m_{i}\right) / m_{i}, \inf _{j} \kappa\left(m_{j}^{\prime}\right) / m_{j}^{\prime}\right\}>0$. Since the convergence in (3) is exponentially fast and therefore summable, we can find $i, j$ such that

$$
\left|\log m_{p}-\log m_{i}-(p-i) v\right|,\left|\log m_{q}^{\prime}-\log m_{j}^{\prime}-(q-j) v^{\prime}\right| \leq \frac{\delta}{150 \alpha^{n}}
$$

for all $p \geq i, q \geq j$. Write $C=\log m_{j}^{\prime}-\log m_{i}$.

Suppose by contradiction that $v / v^{\prime} \notin \mathbb{Q}$. Then there exist integers $p, q>0$ such that $\left|(p-i) v-(q-j) v^{\prime}-C\right| \leq \delta /\left(150 \alpha^{n}\right)$. Combining these statements, we obtain

$$
\left|\log m_{p}-\log m_{q}^{\prime}\right|<\frac{\delta}{50 \alpha^{n}},
$$

Taking the exponential gives

$$
\left|\frac{m_{p}}{m_{q}^{\prime}}-1\right| \leq \frac{\delta}{25 \alpha^{n}},
$$

and therefore

$$
l_{1}=\left|m_{p}-m_{q}^{\prime}\right| \leq \frac{1}{25 \alpha^{n}} \min \left\{\kappa\left(m_{p}\right), \kappa\left(m_{q}^{\prime}\right)\right\} .
$$

It follows that $\varrho$ starts with a $\left\lceil 25 \alpha^{n}\right\rceil$-fold concatenation of the string $\varrho_{1} \ldots$ $\ldots \varrho_{l_{1}}$, or in other words, $\kappa\left(l_{1}\right) \geq 25 \alpha^{n} l_{1}$. Let

$$
l_{2}=\min \left\{\left|\chi^{n}\left(\varrho_{1} \ldots \varrho_{l_{1}}\right)\right| \bmod l_{1}, l_{1}-\left(\left|\chi^{n}\left(\varrho_{1} \ldots \varrho_{l_{1}}\right)\right| \bmod l_{1}\right)\right\} .
$$


We have $l_{2} \neq 0$, because otherwise $\varrho$ is periodic. Since $\left|\chi^{n}\left(\varrho_{1} \ldots \varrho_{l_{1}}\right)\right| \approx$ $\alpha^{n} l_{1} \ll \kappa\left(m_{p}\right)$ and $\kappa\left(m_{q}^{\prime}\right)$, it follows that $\kappa\left(l_{2}\right) \geq 25 \alpha^{n} l_{2}$. Repeating the argument gives a decreasing sequence $\left\{l_{i}\right\}$ of integers such that $\kappa\left(l_{i}\right)>25 \alpha^{n} l_{i}$. In the end also $\kappa(1)$ would be greater than 0 , which is a contradiction, because $\varrho$ does not start with $a_{1} a_{1}$. (More general results on the repetition of words in $\varrho$ were obtained in [12].)

It is straightforward that if $\left\{\widetilde{m}_{i}\right\}$ satisfies (3) for $\widetilde{\varrho}$ and $\widetilde{v}=n \log \widetilde{\alpha}$, then $\left\{m_{i}\right\}$ with $m_{i}=\left|\psi\left(\widetilde{\varrho}_{1} \ldots \widetilde{\varrho}_{\widetilde{m}_{i}}\right)\right|$ satisfies (3) for $\varrho$ and $n \log \widetilde{\alpha}$. Therefore $\log \alpha$ and $\log \widetilde{\alpha}$ are rationally dependent.

COROLlary 2. Let $T_{\alpha}$ be a tent map with an n-periodic critical point. Let $\chi$ and $\varrho$ be as in the proof of Theorem 2. If $n \log \alpha$ is the smallest $v>0$ such that $\varrho$ is asymptotically translation invariant over $v$, then $\left(I, T_{\alpha}\right)$ is not homeomorphic to $\left(I, T_{\widetilde{\alpha}}\right)$ for any $\widetilde{\alpha} \neq \alpha$.

Proof. Suppose by contradiction that $\left(I, T_{\alpha}\right)$ and $\left(I, T_{\widetilde{\alpha}}\right)$ are homeomorphic for $\widetilde{\alpha} \neq \alpha$. Write $T=T_{\alpha}$ and $\widetilde{T}=T_{\widetilde{\alpha}}$. Because $(I, T)$ has exactly $n$ endpoints, $\widetilde{T}$ must have an $n$-periodic critical point as well. From the proof of Theorem 2 (and using a kind of Euclidean dividing algorithm) it follows that there exists $v$ such that $\varrho$ is asymptotically translation invariant over $v$ and that both $n \log \alpha$ and $n \log \widetilde{\alpha}$ are multiples of $v$. By assumption, $v=n \log \alpha$, whence $\log \widetilde{\alpha}$ must be a multiple of $\log \alpha$.

It is well known that if

$$
\frac{1}{m+2} \log 2<\log \alpha \leq \frac{1}{m+1} \log 2,
$$

then $T_{\alpha}$ is renormalizable of period $2^{m}$. By this we mean that there exists an interval $J \ni c$, called a restrictive interval, such that $T^{2^{m}}(J) \subset J$ while $J, T(J), \ldots, T^{2^{m}-1}(J)$ have disjoint interiors. Here the interval $J$ is taken maximal. If $\log \widetilde{\alpha}$ is a multiple of $\log \alpha$, then $\widetilde{T}$ and $T$ must be renormalizable of different periods, say $2^{\widetilde{m}}$ and $2^{m}$, where $m>\widetilde{m}$. Consequently, the critical points of the renormalized maps $\widetilde{T}^{2^{m}}: \widetilde{J} \rightarrow \widetilde{J}$ and $T^{2^{m}}: J \rightarrow J$ have different periods, namely $n / 2^{\widetilde{m}}>n / 2^{m}$. Note that $T^{2^{m}}: J \rightarrow J$ is topologically conjugate to $T_{\alpha^{\left(2^{m}\right)}}:[0,1] \rightarrow[0,1]$. It follows that $(I, T)$ has a subcontinuum

$$
\left\{x \in(I, T): x_{i 2^{m}} \in J \text { for all } i \leq 0\right\}
$$

which is homeomorphic to $\left(I, T_{\alpha^{\left(2^{m}\right)}}\right)$ and therefore has $n / 2^{m}$ endpoints. The space $(I, \widetilde{T})$ has no such subcontinuum.

4. Maps with strictly preperiodic turning points. In this section we conclude the proof of the main theorem by proving 
THEOREM 3. Let $T=T_{\alpha}$ and $\widetilde{T}=T_{\widetilde{\alpha}}$ be tent maps both having a strictly preperiodic critical orbit. If $(\log \alpha) / \log \widetilde{\alpha} \notin \mathbb{Q}$, then the inverse limit spaces $(I, T)$ and $(I, \widetilde{T})$ are not homeomorphic.

The proof is basically the same as the proof of Theorem 2. However, because the turnlink points and the points of the critical orbit are no longer in one-to-one correspondence, we need to be a bit more careful.

Proof of Theorem 3. Let $n=\# \operatorname{orb}_{T}(c)$ and let $c_{2}=y_{1}<\ldots<y_{n}=c_{1}$ be the points of $\operatorname{orb}_{T}(c)$. Let as before $a_{i},-n<i<n, i \neq 0$, denote the intervals $\left[y_{i}, y_{i+1}\right]$ traversed in an orientation preserving (reversing) way if $i>0(i<0)$. By Lemma 2, $C_{t}$ can only turn in $C_{s}$ at links $L \in C_{s}$ such that $\pi_{s}(L) \ni y_{i}$ for some $i$. Therefore the substitution $\chi$ is well defined. Take $y_{i} \in \omega(c)$ and let $N$ be the period of $y_{i}$ if $y_{i}$ is orientation preserving, and twice the period of $y_{i}$ if $y_{i}$ is orientation reversing. Then it is easy to show that for $a \in\left\{a_{-i}, a_{i+1}\right\}, N=\min \left\{k: \chi^{k}(a)\right.$ starts with $\left.a\right\}$.

Define the corresponding notions $\widetilde{n}, \widetilde{y}, \widetilde{\chi}$ and $\widetilde{N}$ for $\widetilde{T}$. Note that in this setting $n$ and $\widetilde{n}$ can be different, but due to Corollary 1 and the remarks following Definition $2, N=\widetilde{N}$.

Assume that $h:(I, T) \rightarrow(I, \widetilde{T})$ is a homeomorphism. By taking $\eta$ small, we can assume that for any set $\widetilde{X} \subset(I, \widetilde{T})$ of diameter $\operatorname{diam}(\widetilde{X})>\delta(\widetilde{T})$, $h^{-1}(\widetilde{X})$ is not contained in a single link of $C_{0}$.

As in the proof of Theorem 2 , take $t_{0}$ so large that $h^{-1}\left(\widetilde{C}_{t_{0}}\right)$ refines $C_{0}$. Each link $\widetilde{L}=\widetilde{L}_{t_{0}, j} \in \widetilde{C}_{t_{0}}$ such that $\widetilde{\pi}_{t_{0}}(\widetilde{L}) \cap \bigcup_{i \geq 1} \widetilde{T}^{i}(c) \neq \emptyset$ is a turnlink. Indeed, if $\widetilde{\pi}_{t_{0}}(\widetilde{L}) \ni \widetilde{T}^{m}(c)$, then $\widetilde{C}_{t_{0}+m}$ turns in $\widetilde{L}$. As $h^{-1}\left(C_{t_{0}}\right)$ refines $C_{0}$, there exists $L \in C_{0}$ such that $h^{-1}\left(\widetilde{L}_{t_{0}, j}\right) \subset L$. We claim that $L$ is a turnlink.

Take $a<j<b$ so that $h^{-1}\left(\bigcup_{k=a+1}^{b-1} \widetilde{L}_{t_{0}, k}\right) \subset L$, but $h^{-1}\left(\widetilde{L}_{t_{0}, a}\right), h^{-1}\left(\widetilde{L}_{t_{0}, b}\right)$ $\not \subset L$. We know that $\widetilde{C}_{t_{0}+m}$ as above turns in $\widetilde{L}_{t_{0}, j}$. Let $a^{\prime}<b^{\prime}$ be such that $\bigcup_{k=a^{\prime}}^{b^{\prime}} \widetilde{L}_{t_{0}+m, k} \subset \bigcup_{k=a}^{b} \widetilde{L}_{t_{0}, k}, h^{-1}\left(\bigcup_{k=a^{\prime}+1}^{b^{\prime}-1} \widetilde{L}_{t_{0}+m, k}\right) \subset L$, and $h^{-1}\left(\widetilde{L}_{t_{0}+m, a^{\prime}}\right)$, $h^{-1}\left(\widetilde{L}_{t_{0}+m, b^{\prime}}\right) \not \subset L$. If $h^{-1}\left(\widetilde{L}_{t_{0}+m, a^{\prime}}\right)$ and $h^{-1}\left(\widetilde{L}_{t_{0}+m, b^{\prime}}\right)$ are contained in the same link $M \in C_{0}$ adjacent to $L$, then $h^{-1}\left(\widetilde{C}_{t_{0}+m}\right)$ turns in $L$ and we are done. If $h^{-1}\left(\widetilde{L}_{t_{0}+m, a^{\prime}}\right)$ and $h^{-1}\left(\widetilde{L}_{t_{0}+m, b^{\prime}}\right)$ are contained in different links of $C_{0}$, then $\bigcup_{k=a^{\prime}+1}^{b^{\prime}-1} \widetilde{L}_{t_{0}+m, k}$ has to turn at least twice in $\bigcup_{k=a}^{b} \widetilde{L}_{t_{0}, k}$, and $\pi_{t_{0}}\left(\bigcup_{k=a^{\prime}+1}^{b^{\prime}-1} \widetilde{L}_{t_{0}+m, k}\right)$ contains at least two different points of $\operatorname{orb}_{\widetilde{T}}(c)$. It follows that $\pi_{0}\left(\bigcup_{k=a^{\prime}+1}^{b^{\prime}-1} \widetilde{L}_{t_{0}+m, k}\right)$ contains two different points of $\operatorname{orb}_{\widetilde{T}}(c)$ and $\operatorname{diam}\left(\bigcup_{k=a^{\prime}+1}^{b^{\prime}-1} \widetilde{L}_{t_{0}+m, k}\right)>\delta(\widetilde{T})$. As $h^{-1}\left(\bigcup_{k=a^{\prime}+1}^{b^{\prime}-1} \widetilde{L}_{t_{0}+m, k}\right) \subset L \in C_{0}$, this contradicts the choice of $\eta$, proving the claim.

By Lemma $2, h^{-1}(\widetilde{L})$ is contained in a link $L \in C_{0}$ such that $\pi_{0}(L) \ni y_{i}$ for some $i \leq n$. Therefore the substitution $\psi$ can be defined properly. By the same arguments, the substitution $\psi_{s, t}$, which expresses how $h\left(C_{t}\right)$ coils 
through $\widetilde{C}_{s}$, can be defined properly for any $t>t_{0}$ and $s>0$ sufficiently large. By taking $t-t_{0}$ and $s$ multiples of $N$ and arbitrarily large, we can derive that $\varrho=\psi(\widetilde{\varrho})$, where $\varrho$ and $\widetilde{\varrho}$ are fixed points of $\chi^{N}$ and $\widetilde{\chi}^{N}$ respectively. The rest of the proof goes through without changes.

Let us finish with some remarks concerning generalizations of the results. We have worked with tent maps for simplicity of the exposition, but the methods work for general unimodal maps just as well. In fact, also the inverse limit spaces of smooth renormalizable maps $f$ can be classified by topological entropy. (It is well known that the entropy is the same for all unimodal maps with the same type of renormalization.) Instead of a finite critical orbit, there is a restrictive interval $J$ whose orbit consists of finitely many (say $n$ ) intervals that are permuted cyclically by $f$. The role of the turnlinks will be taken by clusters of links $\left\{L_{j}\right\}_{j=a}^{b} \subset C_{t}$ such that $\pi_{t}\left(\bigcup_{j=a}^{b} L_{j}\right) \supset f^{i}(J)$ for some $0 \leq i<n$. The rest of the proof remains the same.

Similar results hold for post-critically finite multimodal maps, as well as for piecewise monotone maps on trees whose turning points and branchpoints have finite orbits. We think in particular of Hubbard trees of post-critically finite (quadratic) polynomials [8]. The inverse limit space of a tree map is not chainable because of the branchpoints. Nevertheless, one can define natural covers $C_{t}$ such that every link has at most two neighbours, except for finitely many links for which $\pi_{t}(L)$ contains a branchpoint.

\section{References}

[1] C. Bandt, Composants of the horseshoe, Fund. Math. 144 (1994), 231-241.

[2] M. Barge and B. Diamond, Homeomorphisms of inverse limit spaces of one-dimensional maps, ibid. 146 (1995), 171-187.

[3] M. Barge and S. Holte, Nearly one-dimensional Hénon attractors and inverse limits, Nonlinearity 8 (1995), 29-42.

[4] M. Barge and W. Ingram, Inverse limits on $[0,1]$ using logistic bonding maps, Topology Appl. 72 (1996), 159-172.

[5] M. Barge and J. Martin, Endpoints of inverse limit spaces and dynamics, in: Continua with the Houston Problem Book, Lecture Notes in Pure and Appl. Math. 170, Marcel Dekker, New York, 1995, 165-182.

[6] K. Brucks and B. Diamond, A symbolic representation of inverse limit spaces for a class of unimodal maps, ibid., 207-226.

[7] H. Bruin, Planar embeddings of inverse limit spaces of unimodal maps, Topology Applications 96 (1999), 191-208.

[8] A. Douady et J. Hubbard, Étude dynamique des polynômes complexes, partie I, Publ. Math. Orsay 85-04, 1984.

[9] F. Durand, A generalization of Cobham's Theorem, Theory Comput. Syst. 31 (1998), 169-185.

[10] R. J. Fokkink, The structure of trajectories, Ph.D. thesis, Delft, 1992. 
[11] L. Kailhofer, A partial classification of inverse limit spaces of tent maps with periodic critical points, Ph.D. thesis, Milwaukee, 1999.

[12] B. Mossé, Puissances de mots et reconnaissabilité des points fixes d'une substitution, Theoret. Comput. Sci. 99 (1992), 327-334.

[13] S. Nadler, Continuum Theory, Marcel Dekker, New York, 1992.

[14] M. Queffélec, Substitution Dynamical Systems. Spectral Analysis, Lecture Notes in Math. 1294, Springer, 1987.

[15] R. Swanson and H. Volkmer, Invariants of weak equivalence in primitive matrices, Ergodic Theory Dynam. Systems 20 (2000), 611-616.

[16] W. Watkins, Homeomorphic classification of certain inverse limit spaces with open bonding maps, Pacific J. Math. 103 (1982), 589-601.

Mathematics 253-37

California Institute of Technology

Pasadena, CA 91125, U.S.A.

E-mail: bruin@its.caltech.edu

Received 23 March 1999;

in revised form 7 October 1999 and 11 April 2000 